\title{
The cytoskeletal protein septin 11 is associated with human obesity and is involved in adipocyte lipid storage and metabolism
}

\author{
Natalia Moreno-Castellanos 1,2,3 • Amaia Rodríguez ${ }^{3,4}$ - Yoana Rabanal-Ruiz ${ }^{1,2,3}$ • \\ Alejandro Fernández-Vega ${ }^{1,2,3}$ • José López-Miranda ${ }^{3,6}$ - Rafael Vázquez-Martínez ${ }^{1,2,3}$. \\ Gema Frühbeck $^{3,4,5}$ - María M. Malagón ${ }^{1,2,3}$
}

Received: 11 August 2016 / Accepted: 25 October 2016/Published online: 19 November 2016

(C) Springer-Verlag Berlin Heidelberg 2016

\begin{abstract}
Aims/hypothesis Septins are newly identified members of the cytoskeleton that have been proposed as biomarkers of a number of diseases. However, septins have not been characterised in adipose tissue and their relationship with obesity and insulin resistance remains unknown. Herein, we characterised a member of this family, septin 11 (SEPT11), in human adipose tissue and analysed its potential involvement in the regulation of adipocyte metabolism.

Methods Gene and protein expression levels of SEPT11 were analysed in human adipose tissue. SEPT11 distribution was evaluated by immunocytochemistry, electron microscopy and
\end{abstract}

Electronic supplementary material The online version of this article (doi:10.1007/s00125-016-4155-5) contains peer-reviewed but unedited supplementary material, which is available to authorised users.

María M. Malagón

bc1mapom@uco.es

$\triangle$ Gema Frühbeck

gfruhbeck@unav.es

1 Instituto Maimónides de Investigación Biomédica de Córdoba (IMIBIC)/University of Córdoba/Reina Sofia University Hospital, Edificio IMIBIC, Avda. Menéndez Pidal s/n, 14004 Córdoba, Spain

2 Department of Cell Biology, Physiology and Immunology, University of Cordoba, Córdoba, Spain

3 CIBER Fisiopatología de la Obesidad y Nutrición (CIBEROBN), Instituto de Salud Carlos III, Spain, http://www.ciberobn.es

4 Metabolic Research Laboratory, Clínica Universidad de Navarra, IdiSNA, Pamplona, Spain

5 Department of Endocrinology \& Nutrition, Clínica Universidad de Navarra, Avda. Pío XII 36, 31008 Pamplona, Spain

6 Lipids and Atherosclerosis Unit, IMIBIC/Reina Sofia University Hospital/University of Córdoba, Córdoba, Spain subcellular fractionation techniques. Glutathione $S$-transferase (GST) pull-down, immunoprecipitation and yeast two-hybrid screening were used to identify the SEPT11 interactome. Gene silencing was used to assess the role of SEPT11 in the regulation of insulin signalling and lipid metabolism in adipocytes.

Results We demonstrate the expression of SEPT11 in human adipocytes and its upregulation in obese individuals, with SEPT11 mRNA content positively correlating with variables of insulin resistance in subcutaneous adipose tissue. SEPT11 content was regulated by lipogenic, lipolytic and proinflammatory stimuli in human adipocytes. SEPT11 associated with caveolae in mature adipocytes and interacted with both caveolin-1 and the intracellular fatty acid chaperone, fatty acid binding protein 5 (FABP5). Lipid loading of adipocytes caused the association of the three proteins with the surface of lipid droplets. SEPT11 silencing impaired insulin signalling and insulin-induced lipid accumulation in adipocytes. Conclusions/interpretation Our findings support a role for SEPT11 in lipid traffic and metabolism in adipocytes and open new avenues for research on the control of lipid storage in obesity and insulin resistance.

Keywords Caveolae $\cdot$ FABP5 $\cdot$ Lipid droplets $\cdot$ Lipid metabolism $\cdot$ Obesity $\cdot$ Septins

$\begin{array}{ll}\text { Abbreviations } \\ \% \text { BF } & \text { Percentage body fat } \\ \text { ANP } & \text { Atrial natriuretic peptide } \\ \text { CAV1 } & \text { Caveolin-1 } \\ \text { ERK } & \text { Extracellular signal-regulated kinase } \\ \text { FA } & \text { Fatty acid } \\ \text { FABP5 } & \text { Fatty acid binding protein } 5 \\ \text { GFP } & \text { Green fluorescent protein }\end{array}$




$\begin{array}{ll}\text { GST } & \text { Glutathione } S \text {-transferase } \\ \gamma \text {-GT } & \text { Transaminase glutamyltransferase } \\ \text { IGT } & \text { Impaired glucose tolerance } \\ \text { IR } & \text { Insulin resistance } \\ \text { LPS } & \text { Lipopolysaccharide } \\ \text { LD } & \text { Lipid droplet } \\ \text { NG } & \text { Normoglycaemia } \\ \text { SEPT2 } & \text { Septin 2 } \\ \text { SEPT9 } & \text { Septin 9 } \\ \text { SEPT11 } & \text { Septin 11 } \\ \text { siRNA } & \text { Small interfering RNA } \\ \text { SVFC } & \text { Stromal vascular fraction cells } \\ \text { WAT } & \text { White adipose tissue }\end{array}$

\section{Introduction}

Adipose tissue growth in obesity involves both hypertrophy and hyperplasia of fat cells $[1,2]$. Cytoskeleton remodelling constitutes a prerequisite step for both adipogenesis and adipocyte hypertrophy, increasing the adipocyte lipid storage capability and preventing systemic lipid overload [3-5], which is a major cause of insulin resistance (IR).

The cytoskeletal components actin, tubulin and vimentin undergo important expression and distribution rearrangements during adipogenesis and adipocyte hypertrophy $[3,4,6,7]$. Vimentin forms cage-like structures around adipocyte lipid droplets (LDs) and regulates their accumulation during adipogenesis $[3,7]$. Actin forms cytoplasmic filament bundles and short cortical filaments in pre-adipocytes and mature adipocytes, respectively [3, 4]. A similar reorganisation has been demonstrated for the microtubule network during adipogenesis [3]. A role for actin and tubulin in insulin-induced glucose uptake and lipogenesis in adipocytes has been reported [8,9]. Cortical actin also participates in the maintenance and function of caveolae [10], which are platforms for both insulin signalling and lipid traffic $[11,12]$.

The existence of a fourth component of the cytoskeleton, septins, has been recently proposed [13]. These GTP-binding proteins assemble into filaments and rings and interact with actin and tubulin, profoundly affecting cytoskeletal dynamics, as well as with membrane phospholipids [13]. Septins act as scaffolds for recruiting proteins and as membrane diffusion barriers for subcellular compartmentalisation $[13,14]$. Some septins have been localised to lipid rafts and proposed to regulate membrane dynamics, including endocytosis and exocytosis $[15,16]$.

To date, 13 septin genes have been identified [13]. However, the functional relevance of septins has not yet been described in adipocytes. The present study was focused on septin 11 (SEPT11), which we recently identified in a proteomic screening of human adipose tissue [17]. Specifically, we sought to: (1) characterise the expression and regulation of
SEPT11 in human adipose tissue in relation to obesity and obesity-associated IR and type 2 diabetes; and (2) determine the distribution of SEPT11 and its functional interaction with the main component of caveolae in adipocytes, caveolin-1 (CAV1) [10], and intracellular lipid chaperones.

\section{Methods}

Patient selection and study design Paired omental and subcutaneous adipose tissue samples from patients undergoing laparoscopic Roux-en-Y gastric bypass $(n=54)$ or Nissen funduplication $(n=10)$ at the Clínica Universidad de Navarra (Spain) were obtained. Medical history, physical examination, body composition analysis and comorbidity evaluations were assessed for all patients (see clinical characteristics in Table 1). Obesity was classified according to both BMI ( $\geq 30 \mathrm{~kg} / \mathrm{m}^{2}$ ) and percentage body fat (\%BF; Bod-Pod; Life Measurements, Concord, CA, USA). Obese patients were sub-classified into two groups (normoglycaemia [NG] or impaired glucose tolerance [IGT]/type 2 diabetes] [18]. Obese patients were not using insulin therapy or medication likely to influence endogenous insulin levels and other metabolic variables. Biochemical and hormonal assays were carried out as described [19]. Insulin resistance was calculated using the homeostasis model assessment (HOMA) formula [20], The experimental design was approved by the Hospital's Ethical Committee responsible for research (073/2013) and informed consent was obtained from all volunteers.

RNA extraction and real-time PCR RNA isolation and purification from fat samples were performed as described [19]. Transcript levels of SEPT2, SEPT9, SEPT11, CAV1 and $F A B P 5$ were quantified by real-time PCR (see electronic supplementary material [ESM], and ESM Table 1).

Immunoblotting Tissue and cell samples were processed for immunoblotting as described previously [21]. Blots were incubated overnight at $4{ }^{\circ} \mathrm{C}$ with primary antibodies (see ESM Table 2) and immunoreactive bands were visualised using the ECL Plus detection system (see ESM for further details).

Immunohistochemistry SEPT11 immunodetection in human adipose tissue sections was performed using the immunoperoxidase method [19]. Briefly, sections of formalinfixed paraffin-embedded adipose tissue $(6 \mu \mathrm{m})$ were incubated with rabbit polyclonal anti-SEPT11 antibody (Abcam, Cambridge, UK) and then with Dako Real EnVision horseradish peroxidase (HRP)-conjugated anti-rabbit/mouse (Dako, Glostrup, Denmark). The chromogen was 3,3'-diaminobenzidine and Harris haematoxylin solution was used as counterstaining. See ESM for further details. 
Table 1 Clinical characteristics of the individuals included in the study

\begin{tabular}{lllll}
\hline Characteristic & Lean NG & Obese NG & Obese IGT/T2D & $p$ \\
\hline$n$ & 10 & 24 & 30 & - \\
Sex (male/female) & $3 / 7$ & $12 / 12$ & $14 / 16$ & 0.554 \\
Age (years) & $47 \pm 5$ & $40 \pm 3$ & $44 \pm 1$ & 0.247 \\
Height (m) & $1.64 \pm 0.01$ & $1.69 \pm 0.01$ & $1.67 \pm 0.01$ & 0.323 \\
Weight (kg) & $62 \pm 4$ & $135 \pm 5^{*}$ & $132 \pm 4^{*}$ & $<0.0001$ \\
BMI (kg/m ${ }^{2}$ ) & $22.3 \pm 0.8$ & $47.4 \pm 1.4^{*}$ & $47.6 \pm 1.7^{*}$ & $<0.001$ \\
Body fat (\%) & $26.5 \pm 2.8$ & $51.6 \pm 1.4^{*}$ & $51.6 \pm 1.3^{*}$ & $<0.001$ \\
Waist circumference (cm) & $63 \pm 3$ & $129 \pm 5^{*}$ & $128 \pm 9^{*}$ & 0.001 \\
Glucose (mmol/l) & $4.82 \pm 0.22$ & $4.94 \pm 0.01$ & $6.55 \pm 0.38^{*}$, & 0.002 \\
Glucose 2 h OGTT (mmol/l) & - & $6.49 \pm 0.28$ & $10.38 \pm 0.83^{\dagger}$ & $<0.0001$ \\
Insulin (pmol/l) & $49.2 \pm 10.2$ & $127.8 \pm 19.2^{*}$ & $111.0 \pm 13.2^{*}$ & 0.036 \\
Insulin 2 h OGTT (pmol/l) & - & $591.6 \pm 109.2$ & $516.6 \pm 49.8$ & 0.495 \\
HOMA & $1.75 \pm 0.35$ & $4.86 \pm 0.83^{*}$ & $5.79 \pm 0.98^{*}$ & 0.027 \\
QUICKI & $0.35 \pm 0.01$ & $0.31 \pm 0.01^{*}$ & $0.31 \pm 0.06^{*}$ & 0.030 \\
Triacylglycerol (mmol/l) & $0.8 \pm 0.1$ & $1.3 \pm 0.1$ & $1.9 \pm 0.4$ & 0.292 \\
Total cholesterol (mmol/l) & $5.0 \pm 0.3$ & $5.0 \pm 0.2$ & $5.1 \pm 0.2$ & 0.912 \\
LDL-cholesterol (mmol/l) & $3.1 \pm 0.3$ & $3.2 \pm 0.2$ & $3.3 \pm 0.2$ & 0.771 \\
HDL-cholesterol (mmol/l) & $1.5 \pm 0.1$ & $1.2 \pm 0.1^{*}$ & $1.1 \pm 0.1^{*}$ & 0.027 \\
CRP (mg/l) & $1.8 \pm 0.4$ & $7.7 \pm 1.4^{*}$ & $11.2 \pm 3.1$ & 0.006 \\
Uric acid (mmol/l) & $1.48 \pm 0.17$ & $2.24 \pm 0.13^{*}$ & $2.41 \pm 0.10^{*}$ & 0.003 \\
Leptin (ng/ml) & $7.8 \pm 1.5$ & $46.1 \pm 5.9^{*}$ & $51.2 \pm 6.8^{*}$ & 0.018 \\
AST (U/l) & $13 \pm 3$ & $16 \pm 1$ & $16 \pm 1$ & 0.254 \\
ALT (U/l) & $13 \pm 3$ & $30 \pm 5^{*}$ & $29 \pm 3^{*}$ & 0.039 \\
Alkaline phosphatase (U/1) & $93 \pm 12$ & $113 \pm 7$ & $113 \pm 7$ & 0.576 \\
$\gamma$-GT (U/l) & $9 \pm 2$ & $21 \pm 2$ & $27 \pm 6$ & 0.058 \\
\hline & & & & \\
\hline
\end{tabular}

Differences between groups were analysed by one-way ANOVA followed by Scheffés or $\chi^{2}$ test, where appropriate

$* p<0.05$ vs lean individuals; ${ }^{\dagger} p<0.05$ vs obese NG patients

ALT, alanine aminotransferase; AST, aspartate aminotransferase; CRP, high-sensitivity C-reactive protein
Cell culture Human stromal vascular fraction cells (SVFC) isolated from adipose tissue were differentiated into adipocytes [19] and then exposed to increasing concentrations of insulin, acylated/desacyl-ghrelin, leptin, isoprenaline (known as isoproterenol in the USA), atrial natriuretic peptide (ANP), TNF- $\alpha$, lipopolysaccharide (LPS) and TGF- $\beta$ for $24 \mathrm{~h}$, or with oleate complexed to BSA $(2: 1)$ for $18 \mathrm{~h}$. 3T3-L1 cells were differentiated as described $[21,22]$ and exposed to oleate for $18 \mathrm{~h}$ (see ESM for further details).

Confocal immunofluorescence microscopy Freshly isolated mature adipocytes, in vitro differentiated human adipocytes and 3T3-L1 cells grown on glass coverslips were fixed in $4 \%$ paraformaldehyde as described earlier [21, 22]. Cells were immunostained using anti-SEPT11 (1:100) (gift from M. Kinoshita, Nagoya University, Japan) and/or anti-CAV1 (1:250) (Novus Biologicals, Littleton, CO, USA) antibodies, and examined by confocal microscopy (see ESM for further details).
Immunoelectron microscopy Ultrathin sections of 3T3-L1 adipocytes were incubated with anti-SEPT11 and $10 \mathrm{~nm}$ gold conjugated anti-rabbit IgG, and visualised using a JEOL JEM2010 electron microscope (IZASA Scientific, Madrid, Spain) [23].

Subcellular fractionation Cytosolic and crude membrane fractions were isolated from 3T3-L1 adipocytes as previously described [24]. Caveolae-enriched membrane fractions were isolated from 3T3-L1 cells grown to confluency and processed using a detergent-free cell membrane fractionation method (see ESM for further details) [24]. Protein distribution was analysed by immunoblotting using $50 \mu \mathrm{g}$ of protein from each fraction (see antibodies in ESM Table 2).

Plasmid expression vectors SEPT11 was cloned in DsRed-, pGEX-4T-3-, Myc- and phrGFP-N1 expression vectors. Plasmids coding for septin 2 (SEPT2; phrGFPN1-SEPT2), septin 9 (SEPT9; pEGFPC1-SEPT9), fatty acid binding 
protein 5 (FABP5; pEGFPC1-FABP5) and CAV1 (CFP-CAV1 [24]) were also employed (see ESM for further details).

Yeast two-hybrid screening The SEPT11 interactome was identified by a yeast two-hybrid method using a rat subcutaneous adipose tissue cDNA library with SEPT11 as bait and the Matchmaker Gold Yeast Two-Hybrid System (Clontech; Mountain View, CA, USA) (see ESM for further details). The rat subcutaneous adipose tissue cDNA library was constructed according to the manufacturer's instructions (Make Your Own 'Mate \& Plate' Library System, Clontech). Vectors containing the yeast GAL4 binding domain, with or without the insert for SEPT11, were individually cotransformed into yeast Y2HGold with a vector containing the GAL4 activation domain fused with the library plasmid. Positive interactions were detected and analysed as described in ESM.

Glutathione $S$-transferase pull-down and co-immunoprecipitation assays The glutathione $S$-transferase (GST) protocol was performed using GST-tagged SEPT11 and phrGFPN1-SEPT2, pEGFPC1-SEPT9, pEGFPC1-FABP5 or CFP-CAV1. A standard protocol was used for co-immunoprecipitation of SEPT11 and CAV1 in HEK293 AD cells transfected with Myc-SEPT11 and CFP-CAV1 (see ESM for further details).

Electroporation of 3T3-L1 adipocytes Differentiated 3T3$\mathrm{L} 1$ adipocytes were transfected by electroporation with mock vectors, phrGFPN1-SEPT2, pEGFPC1-SEPT9 or DsRedSEPT11, alone or in combination, and cultured for $48 \mathrm{~h}$ [22]. 3T3-L1 adipocytes transiently expressing pEGFPC1-FABP5 were incubated with $500 \mu \mathrm{mol} / 1$ oleate:BSA $(2: 1)(18 \mathrm{~h})$ and then double-immunolabelled with anti-CAV1 and antiSEPT11 antibodies.

For silencing studies, SEPT11-targeted double-stranded small interfering (si)RNA oligonucleotide and a specific scramble negative control siRNA (Qiagen, Barcelona, Spain) were employed for electroporation. Insulin signalling (pAkt/Akt, p-extracellular signal regulated kinase (ERK)/ ERK, pIRS/IRS) was examined in Sept11-silenced 3T3-L1 cells challenged with insulin ( $100 \mathrm{nmol} / 1,5 \mathrm{~min})$ by immunoblotting. Intracellular triacylglycerols and glycerol in culture media were quantified in Sept11-silenced and mocktransfected 3T3-L1 cells exposed to insulin $(100 \mathrm{nmol} / \mathrm{l})$ or insulin plus oleate $(500 \mu \mathrm{mol} / \mathrm{l})$, as previously described [22] (see ESM for further details).

Statistical analysis Data are expressed as mean \pm SEM. Statistical differences were determined using the $\chi^{2}$ test, two-way or one-way ANOVA followed by Scheffé's or Dunnett's test, or Kruskal-Wallis test followed by MannWhitney $U$ pairwise comparisons. Values were considered significant at $p<0.05$. Pearson's correlation coefficients $(r)$ were computed to explore the relation between two variables.

\section{Results}

Characterisation of SEPT11 in human adipose tissue SEPT11 was expressed in omental and subcutaneous fat, with the former depot exhibiting higher overall transcript levels $(1.0 \pm 0.0$ vs $5.7 \pm 1.3 \mathrm{AU}, p<0.0001)$. Omental and subcutaneous adipose tissue from obese patients showed higher SEPT11 mRNA and protein than lean individuals (Fig. 1ad); no differences were found between NG and IGT/type 2 diabetic obese patients. SEPT11 protein levels in omental fat were positively related to markers of adiposity (BMI $[r=0.45$, $p=0.010], \% \mathrm{BF}[r=0.47, p=0.010]$, and plasma leptin $[r=0.38, p=0.049])$, while positively associated with variables of glucose metabolism and hepatic function in subcutaneous fat (glycaemia $[r=0.80, p<0.001]$, insulinaemia $[r=0.44, p=0.030]$, HOMA $[r=0.69, p<0.001]$, QUICKI $[r=-0.47, p=0.018]$, serum triacylglycerol $[r=0.82$, $p<0.001]$ and transaminase glutamyltransferase $[\gamma-\mathrm{GT}]$ $[r=0.71, p<0.001])$.

The SEPT11 immunosignal mostly localised to mature adipocytes and, to a lesser extent, SVFC in omental and subcutaneous fat (Fig. 1e, f). Similar results were observed for SEPT11 mRNA in adipocytes and SVFC from omental adipose tissue of obese patients (Fig. 1g). Insulin-sensitive and insulin-resistant obese patients exhibited similar SEPT11 mRNA levels in either adipocytes or SVFC from omental fat (Fig. 1h). The transcript levels of the pre-adipocyte marker, PREF1 (also known as DLK1), and the macrophage marker, $C D 68$, were analysed to determine the contribution of these cell types to the overall expression in SVFC of adipose tissue (ESM Fig. 1). Lower PREF1 mRNA expression as well as higher $C D 68$ transcript levels were observed in omental SVFC from obese IGT/type 2 diabetic vs obese NG individuals, although the differences did not reach statistical significance ( $p=0.144$ and $p=0.459$, respectively). No correlation was found between SEPT11 transcripts and PREF1 $(r=0.08$, $p=0.865)$ or $C D 68(r=0.30, p=0.326)$, suggesting that other cell types may contribute to SEPT11 gene expression in SVFC. Indeed, SEPT11 is reportedly expressed by other stromal vascular fraction components (fibroblasts, neutrophils) $[25,26]$.

Regulation of SEPT11 in human omental adipocytes We evaluated the effect of lipogenic (insulin, ghrelin), lipolytic (leptin, isoprenaline, ANP) and proinflammatory factors (TNF- $\alpha$, LPS, TGF- $\beta$ ) on SEPT11 in human omental adipocytes. Insulin significantly increased SEPT11 mRNA and protein, while acylated ghrelin downregulated SEPT11 mRNA and desacyl-ghrelin had no effect (Fig. 2a, b). Isoprenaline 
Fig. 1 Characterisation of SEPT11 in human adipose tissue. SEPT11 mRNA and protein and tissue distribution in omental (a, $\mathbf{c}, \mathbf{e})$ and subcutaneous $(\mathbf{b}, \mathbf{d}, \mathbf{f})$ white adipose tissue (WAT) of lean participants, obese NG patients, and obese IGT and type 2 diabetic patients (scale bars, $50 \mu \mathrm{m}$ ). Representative images of three separate experiments are shown. No immunoreactivity was found without primary antibody (control). Comparison of SEPT11 transcripts in freshly isolated adipocytes and SVFC from omental WAT of obese individuals globally $(n=10)(\mathrm{g})$ and separated according to IR $(n=9)(\mathbf{h})$ : white bars, obese NG; black bars, obese IGT/type 2 diabetic. $* p<0.05,{ }^{*} p<0.01$ and $* * * p<0.001$ vs lean NG or control adipocytes. ${ }^{\dagger \dagger} p<0.001$ vs adipocytes. T2D, type 2 diabetic a

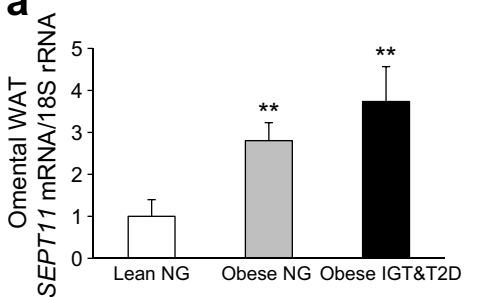

C
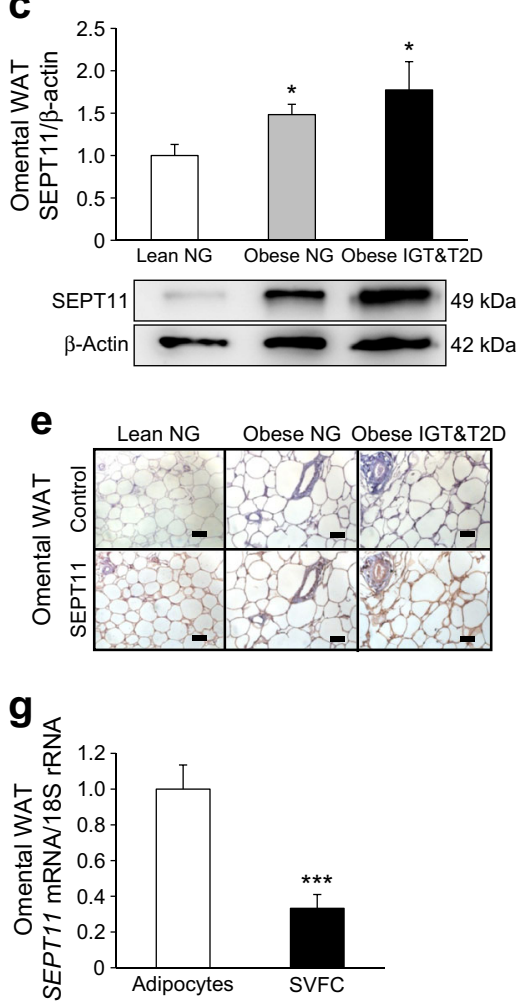

b
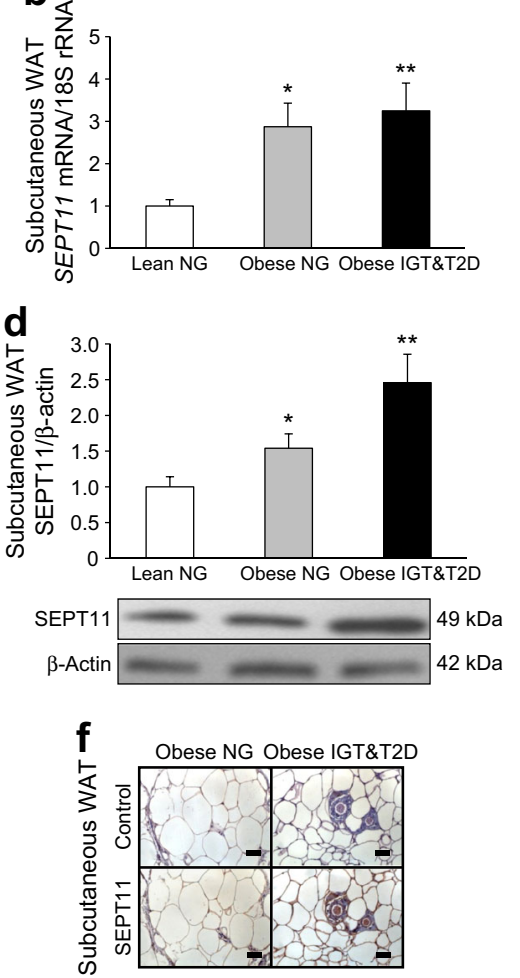

$\mathbf{h}_{\triangle}$

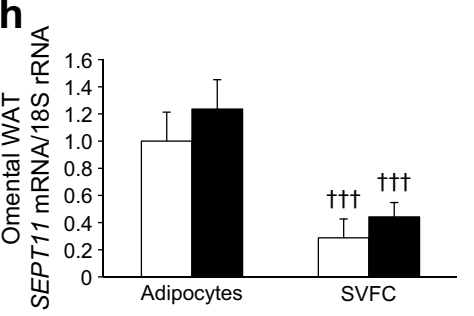

and ANP downregulated SEPT11 mRNA and protein, although statistical differences were only reached for isoprenaline (Fig. 2c, d). TNF- $\alpha$ and LPS downregulated SEPT11 mRNA and protein, while leptin and TGF- $\beta$ had no effect (Fig. 2d-f).

SEPT11 distribution in adipocytes and interaction with other septins Immunocytochemistry confocal microscopy studies revealed that SEPT11 associated with cytosolic filamentous structures reminiscent of actin stress fibres in undifferentiated (3 days) 3T3-L1 cells, while it associated with cell surface ring-like structures in differentiated 3T3-L1 adipocytes (Fig. 3a). Similar results were observed in human adipocytes (ESM Fig. 2a). Given that septin filaments and rings are composed of heteroligomeric septin complexes, we examined the relationship between SEPT11 and other septins known to interact with SEPT11, SEPT9 and SEPT2 [25, 27]. Contrary to endogenous SEPT11, green fluorescent protein (GFP)-SEPT11 formed short fibres when transfected alone in 3T3-L1 adipocytes (ESM Fig. 3b). However, when co-expressed, GFP-SEPT11 and GFP-SEPT2 formed short fibres and rings, while GFP-SEPT11 and GFP-SEPT9 assembled into long filaments (Fig. 3c). SEPT11 association with SEPT2 and SEPT9 was confirmed by GST pull-down (Fig. 3d).

SEPT2 was expressed in omental and subcutaneous fat, with the three groups of patients showing similar SEPT2 transcript levels in these depots (Fig. 3e, f). SEPT2 mRNA in omental fat positively associated with insulinaemia $(r=0.34$, $p=0.027)$, while in subcutaneous fat a positive correlation with SEPT11 $(r=0.39, p=0.005)$ and SEPT9 $(r=0.61$, $p<0.0001)$ transcripts was found, yet only the latter remained after adjustment for \%BF ( $r=0.42, p=0.005)$. SEPT9 mRNA in omental fat was increased in obese IGT/type 2 diabetic patients compared with lean and obese NG participants, while SEPT9 mRNA in subcutaneous fat decreased in both obese groups (Fig. 3e, f). SEPT9 transcripts positively correlated with SEPT11 expression $(r=0.50, p=0.005)$ in omental fat and with plasma glucose with 2 h OGTT $(r=0.47$, $p=0.004$ ), before adjustment for $\% \mathrm{BF}$. 
Fig. 2 Regulation of SEPT11 in human omental adipocytes. Effect of lipogenic $(\mathbf{a}, \mathbf{b})$, lipolytic $(\mathbf{c}, \mathbf{d})$ and proinflammatory $(\mathbf{e}, \mathbf{f})$ stimuli on SEPT11 mRNA and protein in human omental adipocytes ( $n=8-12$ per group). $* p<0.05$ and $* * p<0.01$ vs unstimulated adipocytes

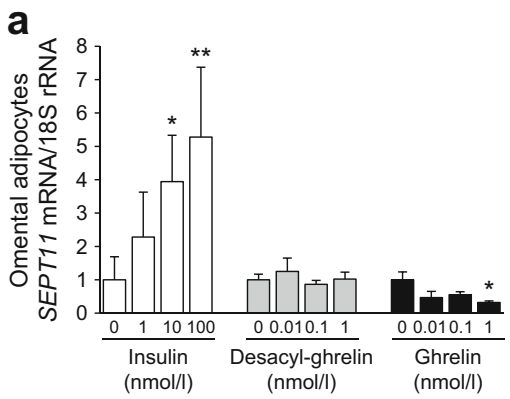

b
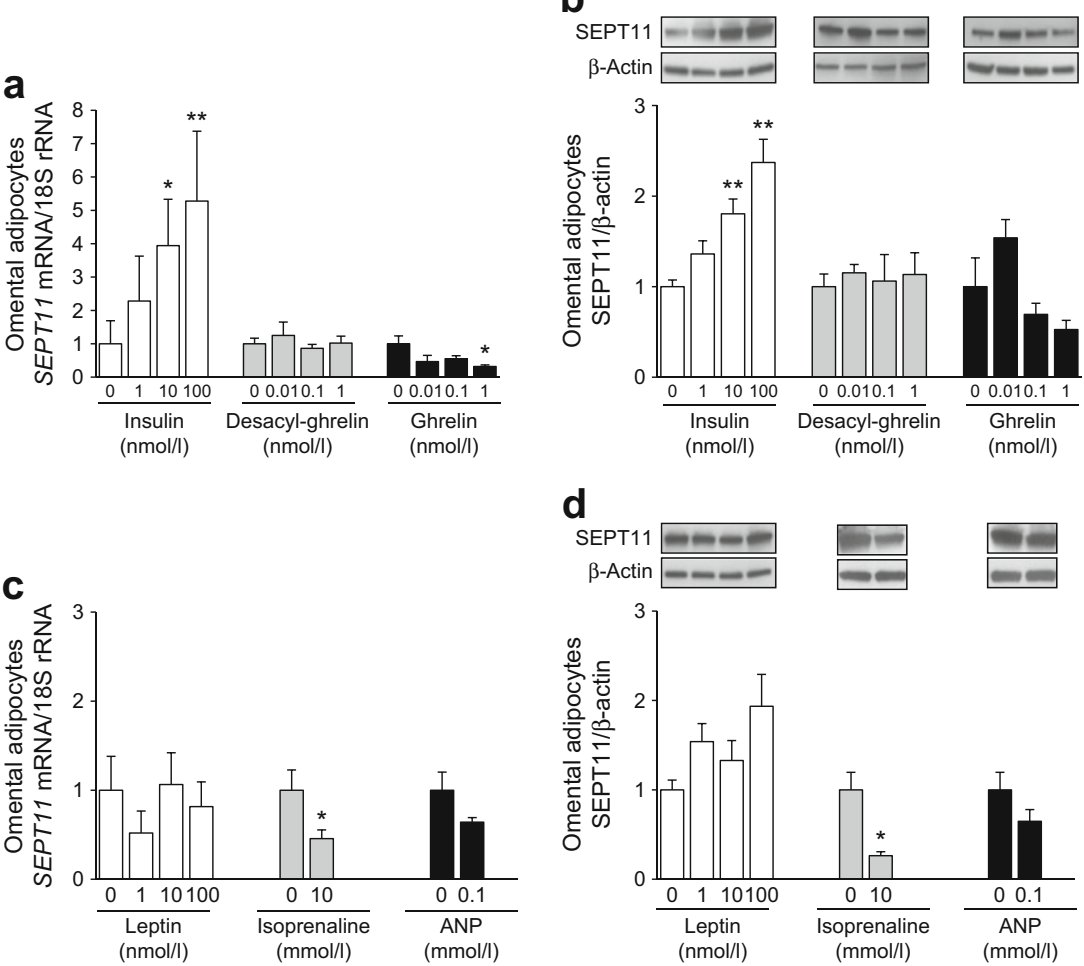

d
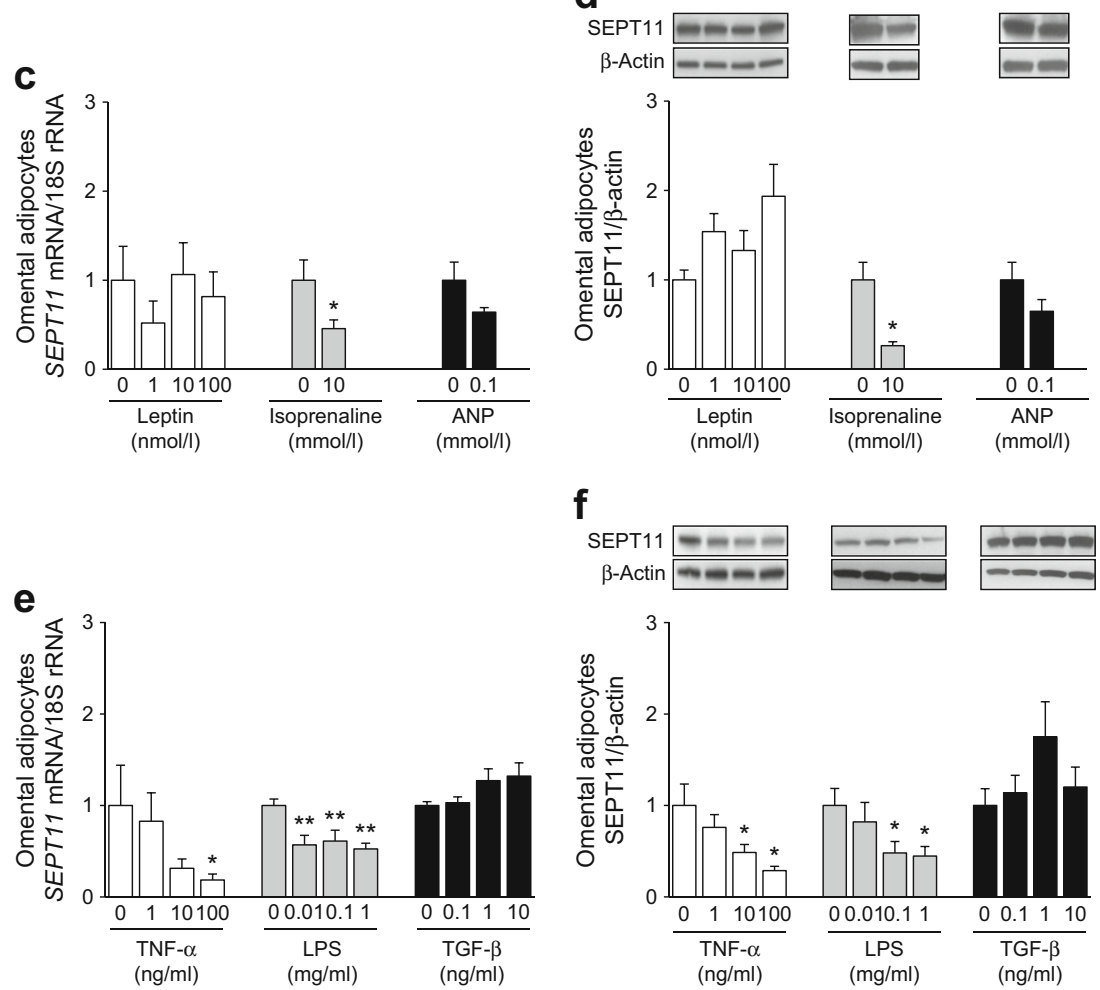

Localisation of SEPT11 to adipocyte caveolae SEPT11immunoreactive rings at the adipocyte surface (Fig. 3a) resembled the ring-like shape of caveolae clusters reported in adipocytes [28]. Indeed, SEPT11 and CAV1 immunosignals significantly overlapped in cortical rings in freshly isolated human mature adipocytes (Fig. 4a) and 3T3-L1 adipocytes (ESM Fig. 3a). Immunoelectron microscopy of 3T3-L1 adipocytes showed SEPT11 immunolabelling associated with plasma membrane invaginations resembling caveolae (Fig. 4b). Subcellular fractionation of 3T3-L1 adipocytes revealed that SEPT11 mostly associated with cellular membranes (Fig. 4c). Analysis of isolated caveolae-enriched membrane [24] showed that SEPT11 immunoreactivity was essentially associated with non-buoyant fractions (Fig. 4d). This indicated that SEPT11 interacts with caveolae as a peripheral protein, which was further demonstrated by immunoblotting of membrane fractions from 3T3-L1 adipocytes treated with compounds known to differentially extract peripheral and integral membrane proteins (ESM Fig. 3b).

GST-SEPT11 pull-down (Fig. 4e) and immunoprecipitation assays (ESM Fig. 3c) confirmed the association of SEPT11 with CAV1. CAV1 interaction with septins was also investigated in human adipose tissue (Fig. 4f). CAV1 mRNA expression in omental fat was increased in obese compared with lean individuals, though no differences were found between NG and IGT/type 2 diabetic obese participants. A tendency towards an increase in CAV1 mRNA in obese subcutaneous fat was observed $(p=0.290)$. CAV1 mRNA expression positively correlated with SEPT11 $(r=0.39, p=0.006)$ and SEPT9 $(r=0.48, p<0.0001)$ transcripts in omental fat (Fig. 4g; ESM Fig. 4c) and with SEPT11 $(r=0.41$, $p=0.001), \operatorname{SEPT2}(r=0.48, p<0.0001)$ and SEPT9 
Fig. 3 Distribution of SEPT11 in adipocytes and interaction with other septins. (a) Confocal microscope images of SEPT11 immunosignal in undifferentiated (left) and differentiated (right) 3T3-L1 adipocytes. (scale bars, $5 \mu \mathrm{m})$ (b) 3T3-L1 adipocytes overexpressing SEPT2 and SEPT9 alone (green), or (c) in combination with SEPT11 (red). Colocalisation of the septin pairs is shown in yellow. (scale bars,

$10 \mu \mathrm{m}$ ). Representative images of at least three separate experiments are shown. (d) Direct interaction between SEPT11 with SEPT2 and SEPT9 revealed by GST pulldown. Input and pull-down samples (IP, immunoprecipitate) were subjected to immunoblotting with antiSEPT11 and anti-GFP antibodies. Gene expression of SEPT2 and SEPTY in omental (e) and subcutaneous (f) WAT of lean (white bars), obese NG (grey bars) and IGT/type 2 diabetic (black bars) individuals. $* p<0.05$ and $* * p<0.01$ vs lean NG participants. ${ }^{\dagger \dagger} p<0.01$ vs obese NG participants a
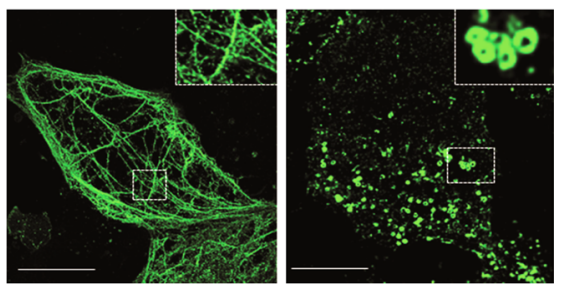

d

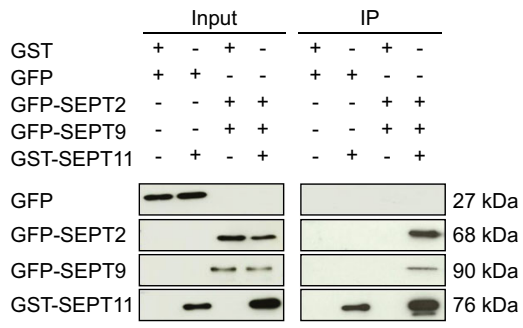

b

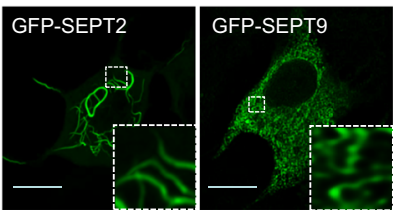

C

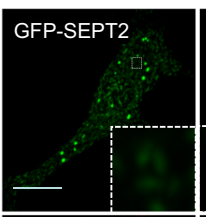

GFP-SEPT9

SEPT11-DsRed

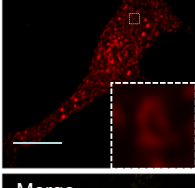

SEPT11-DsRed

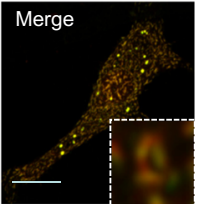

.
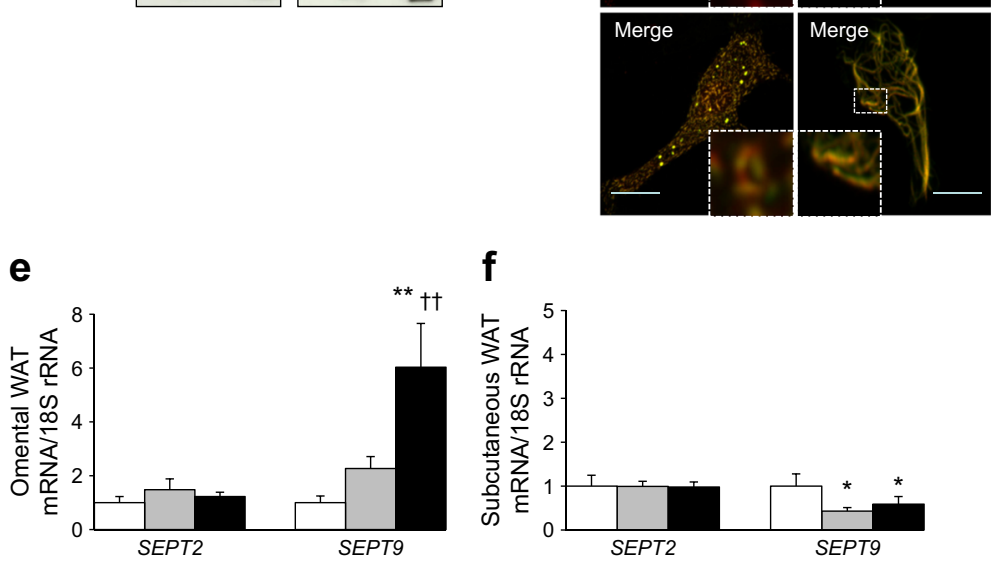

f

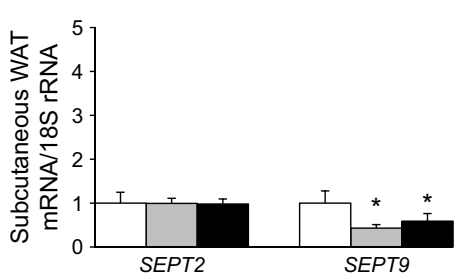

$(r=0.39, p=0.005)$ in subcutaneous fat (Fig. 4h; ESM Figs. 4b, d).

\section{SEPT11 binds to FABP5 and regulates adipocyte lipid} metabolism To elucidate SEPT11 function, we examined its interactome by yeast two-hybrid screening of a rat adipose tissue cDNA library using SEPT11 as bait. This enabled the identification of 15 putative SEPT11 interactors (ESM Table 3). Five positive clones encoded the fatty acid (FA) chaperone FABP5, and SEPT11 interaction was confirmed by GST pull-down (Fig. 5a). Quantitative RT-PCR revealed lower FABP5 transcript levels in omental fat of obese patients compared with lean individuals (Fig. 5b).

To further examine the interaction between FABP5 and SEPT11, we investigated FABP5 distribution in adipocytes. When expressed in 3T3-L1 adipocytes, GFP-FABP5 mostly distributed diffusely in the cytosol, though some labelling concentrated beneath the plasma membrane (Fig. 5c). Exposure of cells to oleate promoted FABP5 association with LDs and nuclei. CAV1 and SEPT11 also associated with LDs in oleate-stimulated 3T3-L1 (Fig. 5c) and human (ESM Fig. 5) adipocytes. Triple-labelling experiments showed that while GFP-FABP5 occasionally co-localised with CAV1 and SEPT11 in non-stimulated 3T3-L1 cells, the three fluorescent labels overlapped around LDs in oleate-treated adipocytes (Fig. 5c). Oleate increased SEPT11 content by 4.1-fold without modifying CAV1 and FABP5 levels in adipocytes (Fig. 5d).

Next, the potential role of SEPT11 in the regulation of known caveolae-associated functions in adipocytes, namely insulin signalling and lipid regulation, was determined in 3T3-L1 cells treated with Sept11 siRNA, which reduced by $71.1 \%$ SEPT11 protein content compared with scrambled siRNA-treated cells (Fig. 5e), without compromising cell viability (ESM Fig. 6a). SEPT11 silencing also reduced CAV1 and FABP5 content (Fig. 5e), but not that of other adipocyte markers (ESM Fig. 6b). Regarding insulin signalling, Sept11-silenced adipocytes showed significant reductions in total IRS and pAkt/Akt ratio as well as a trend towards decreased pIRS/IRS ratio (Fig. 5f). No effect on ERK content or activity was observed. SEPT11 depletion tended to decrease basal and insulin-induced lipogenesis (Fig. 5g). Notably, insulinstimulated intracellular triacylglycerol accumulation in the presence of oleate was reduced by $31 \%$ in Sept11-silenced 
Fig. 4 Caveolar localisation of SEPT11 in adipose tissue. (a) Colocalisation of SEPT11 and CAV1 in mature adipocytes (scale bars, $10 \mu \mathrm{m}$ ). (b) Electron microscope image of SEPT11 showing a caveolar localisation $(\times 71,000$; scale bar, $200 \mathrm{~nm})$. Representative images of three separate experiments are shown. (c) SEPT11 and CAV1 protein expression in total, cytosolic and membrane fractions. GM130 and $\beta$-actin were used as membrane and cytosolic markers, respectively. (d) SEPT11 and CAV1 distribution in caveolaeenriched membranes from 3T3L1 cells. (e) Direct interaction between SEPT11 and CAV1 revealed by GST pull-down. (f) CAV1 mRNA expression and its correlation with SEPT11 transcripts $(\mathbf{g}, \mathbf{h})$ in paired omental $(r=0.39, p=0.006)$ and subcutaneous $(r=0.41, p=0.001)$ WAT obtained from lean (white circles), obese NG (grey circles) and IGT/type 2 diabetic (black circles) participants. $* * p<0.01$ vs lean volunteers. $\mathrm{C}$, cytosolic fraction; IP, immunoprecipitate; $\mathrm{M}$, membrane fraction; TF, total fraction a
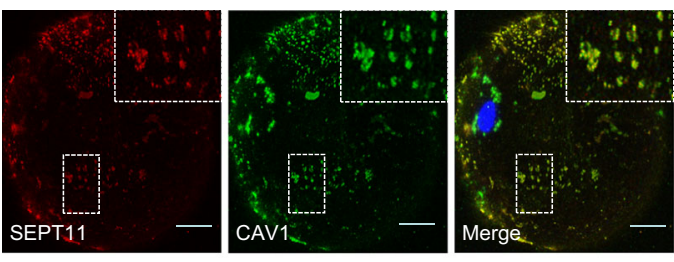

b

C

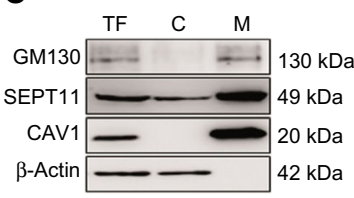

d

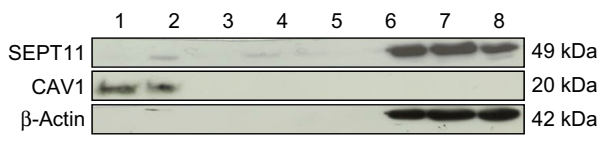

e
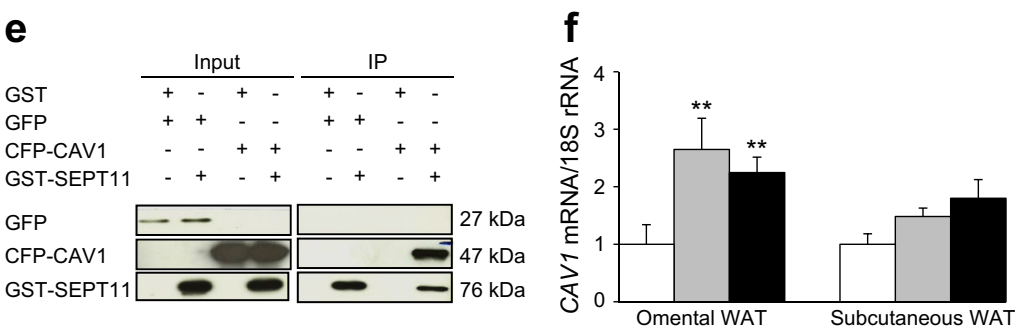

g

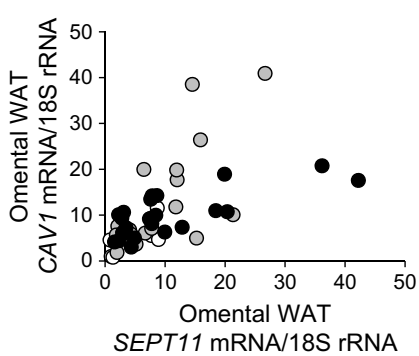

h

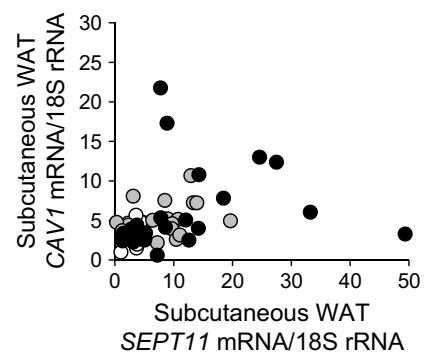

adipocytes (Fig. 5g). SEPT11 silencing also diminished the lipolytic rate of 3T3-L1 adipocytes under all the experimental conditions tested (ESM Fig. 7).

\section{Discussion}

Our group recently reported the expression of SEPT11 in human adipose tissue [17]. Herein, we demonstrate that SEPT11 is expressed primarily in adipocytes of human adipose tissue. Moreover, a higher expression of SEPT11 was found in subcutaneous fat compared with omental fat. It is well known that visceral fat shows higher inflammation and sensitivity to catecholamines, while subcutaneous fat exhibits higher insulin sensitivity [29]. We show that SEPT11 was negatively regulated by isoprenaline, TNF- $\alpha$ and LPS, while positively regulated by insulin. Accordingly, SEPT11 expression was positively related to markers of adiposity in omental fat and with variables of IR in subcutaneous fat. Altogether, fat depot differences in SEPT11 expression appear to be associated with higher IR, lipolysis and inflammation in omental adipose tissue.
Obesity was associated with higher SEPT11 levels in omental and subcutaneous fat, which might be accounted for by increased SEPT11 in mature adipocytes as well as in inflammatory cells of the SVFC, as suggested by our observations and previous studies showing the presence of this protein in macrophage and neutrophil cell lines $[25,26]$. Notably, no differences were found between normoglycaemic and insulinresistant obese individuals, suggesting that the regulation of SEPT11 may be related to adipocyte size rather than to IR. In line with this, SEPT11 protein levels in 3T3-L1 and human adipocytes increased after exposure to the lipogenic factors, oleate and insulin. Moreover, adipogenesis was associated in both cell types with an extensive reorganisation of SEPT11, i.e. from long linear bundles resembling actin stress fibres to submembranous structures. The actin cytoskeleton undergoes similar rearrangements during adipogenesis [5], supporting a functional interaction between SEPT11 and actin in adipocytes similar to that reported for several septins in other cell types [25, 30-32].

All septins interact with each other to form heteroligomeric complexes, with changes in septin-septin interaction causing 
Fig. 5 SEPT11 regulates lipid metabolism targeting FABP5 in adipocytes. (a) Direct interaction between SEPT11 and FABP5 revealed by GST pull-down. (b) FABP5 mRNA expression in omental and subcutaneous WAT of lean, obese NG and IGT/type 2 diabetic patients. (c) Colocalisation studies of SEPT11 (red), CAV1 (blue), GFP-FABP5 (green) in unstimulated (basal) or oleate-treated 3T3-L1 cells $(500 \mu \mathrm{mol} / \mathrm{l}, 18 \mathrm{~h})$ (scale bars, $10 \mu \mathrm{m})$. Representative images of at least three separate experiments are shown. Protein levels of SEPT11, CAV1 and FABP5 after $18 \mathrm{~h}$ oleate treatment (d) or Sept11 gene silencing (e). Insulin signalling intermediates (f) in SEPT11-knockdown 3T3-L1 adipocytes. (g) Intracellular triacylglycerol content in control and Sept11-silenced 3T3-L1 adipocytes under basal conditions (white bars) and $18 \mathrm{~h}$ after treatment with insulin (grey bars) or oleate plus insulin (black bars). Values are the mean \pm SEM $(n=6) . * p<0.05, * * p<0.01$ and $* * * p<0.001$ vs unstimulated control cells $(100 \%) ;{ }^{\dagger \dagger} p<0.01$ and ${ }^{\dagger \dagger} p<0.001$ vs control siRNA cells with same stimuli. IP, immunoprecipitate; TG, triacylglycerol

\section{a}

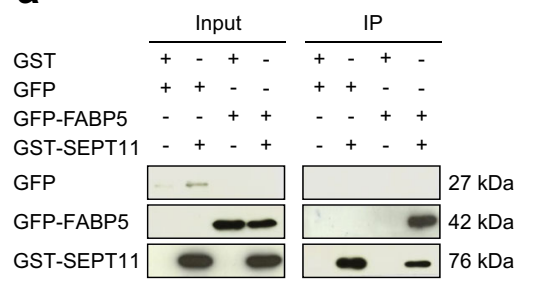

b

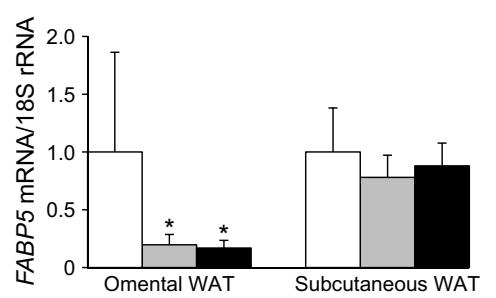

C
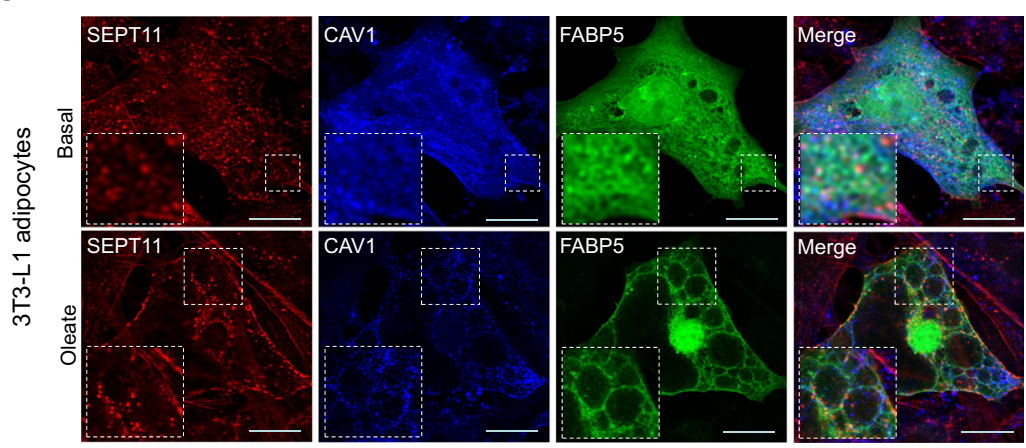

d

e

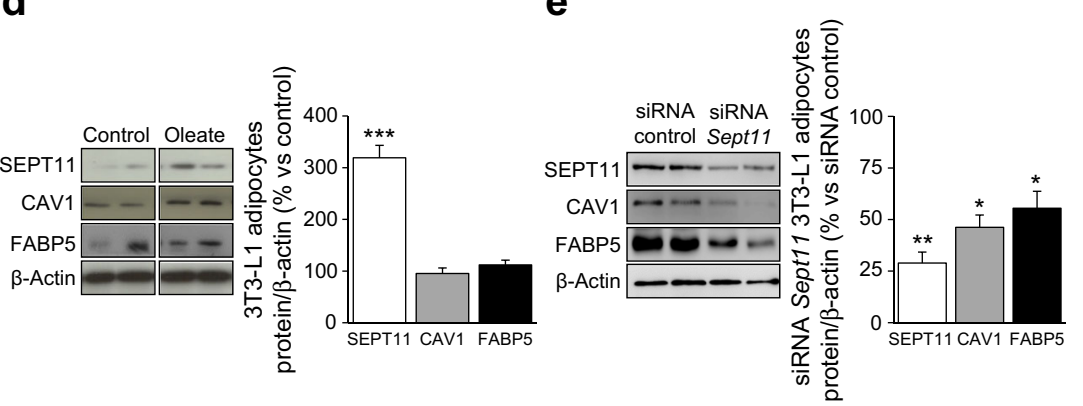

f
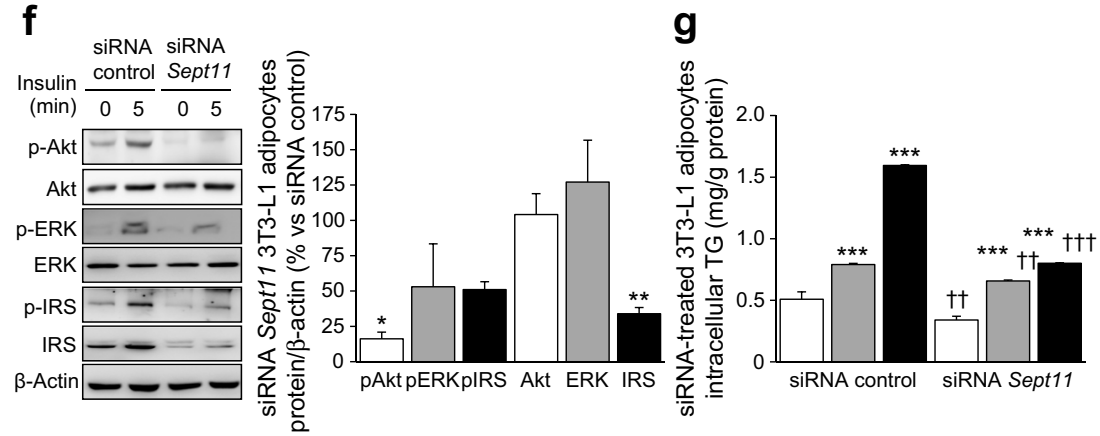

changes in structural conformation and function of the complex [27, 33, 34]. Specifically, previous studies have described the association of SEPT11 with SEPT2 or SEPT9 [27, 34]. Herein, the expression of SEPT2 and SEPT9 in human fat and their direct interaction with SEPT11 was demonstrated. Notably, SEPT11 formed short filaments when expressed alone in adipocytes, while it assembled into rings or long filaments when co-expressed with SEPT2 or SEPT9, respectively. These findings suggest that the filament and ring-like assemblies observed for SEPT11 may be composed of complexes of this protein with SEPT2 and/or SEPT9. In contrast to what was seen with SEPT11, obesity was associated with increased expression of SEPT9 in omental fat and low SEPT9 mRNA levels in subcutaneous fat, without changes in SEPT2 in either depot, suggesting a distinct adaptation of these septins to adipocyte hypertrophy and IR. Changes in the stoichiometry of septin complexes have been associated with several diseases [35]. It seems plausible that the dissimilar changes in SEPT2, SEPT9 and SEPT11 transcription in the obese state alter normal SEPT11-containing polymer structures and functions. 
Cortical SEPT11-immunoreactive rings were observed in 3T3-L1 adipocytes and in freshly isolated and in vitro differentiated human adipocytes, indicating that this oligomeric complex is the main functional unit in mature fat cells. Confocal microscopy studies revealed a significant overlap of SEPT11 and CAV1 immunosignals at the cell surface which, together with the morphological resemblance of SEPT11 rings to caveolae clusters in adipocytes [28] and our immunoelectron microscopy data, demonstrate that this protein associates with adipocyte caveolae. Further support for this proposal has been provided by our biochemical studies showing that SEPT11 physically interacts with CAV1. Interestingly, a positive correlation of CAV1 with SEPT11 expression was observed in human adipose tissue. In all, our studies suggest a role for SEPT11 in the regulation of caveolae function, which we further explored through siRNA-induced SEPT11 silencing. Interestingly, SEPT11 silencing in 3T3-L1 adipocytes, besides decreasing CAV1 content, significantly diminished insulinstimulated Akt phosphorylation, indicating impaired insulin signalling under conditions of diminished SEPT11 expression (Fig. 6). Accordingly, pAkt levels are greatly reduced in Cav1-null mouse adipose tissue compared with wildtype controls [36]. SEPT11 seems to differentially modulate specific insulin-mediated kinase pathways as the loss of this protein did not apparently affect insulin-stimulated ERK signalling. Nevertheless, we cannot exclude the possibility that the residual SEPT11 and/or partial septin complexes lacking SEPT11 are sufficient to support normal ERK signalling.

Several mechanistic reports indicate a role for septins as diffusion barriers that help to compartmentalise cellular membranes into separate domains $[13,33,34]$. In this vein, SEPT11 association with caveolae could help maintain the compartmentalisation and function of these microdomains in the regulation of signalling cascades [11, 37]. Septins have also been shown to serve as subcellular scaffolds for protein recruitment [13]. Notably, we identified FABP5 as a SEPT11 partner by a yeast two-hybrid assay. FABP5, together with fatty acid binding protein 4 (FABP4), are the main FA-binding proteins in adipose tissue [38]. FA-binding proteins have been proposed to mediate FA uptake and to facilitate lipid transport to LDs [38-40]. Accordingly, we observed that GFP-FABP5 mainly exhibited a diffuse cytoplasmic distribution in non-stimulated adipocytes, although it accumulated more densely beneath the plasma membrane. Exposure of cells to oleate, which binds with high affinity to FABP5 [41], induced the association of FABP5 and SEPT11 with the LD surface. In agreement with previous reports [11], we observed a similar traffic route for CAV1 in response to FA loading. Moreover, triple-labelling microscopy studies showed that SEPT11 co-localised with CAV1 and GFP-FABP5 to LDs in oleate-treated cells. Taken together, these results indicate that the CAV1/SEPT11 interaction extends from caveolae to LDs, wherein SEPT11 presumably also associates with FABP5. It has been proposed that caveolins at caveolae serve to modulate transmembrane FA movement [42], whereas the LD caveolin pool might play a role in LD expandability [37, 43]. Specifically, oleate-loaded fibroblasts from Cav1-null mice stored fewer and smaller LDs than control cells, suggesting that CAV1 facilitates LD accumulation [37]. Interestingly, SEPT11 knockdown led to a decrease in insulin-induced lipid accumulation, which is consistent with our observations of
Fig. 6 Proposed working model for the role of SEPT11 in insulin signalling and lipid traffic in the caveolae. SEPT11 constitutes a cytoskeletal protein that forms heterocomplexes with SEPT2 and SEPT9 and is transcriptionally regulated by insulin. SEPT11 locates to the caveolae of adipocytes and, on lipid loading, associates with CAV1 and FABP5 at the surface of LDs contributing to lipid traffic. PI3K, phosphoinositide 3-kinase

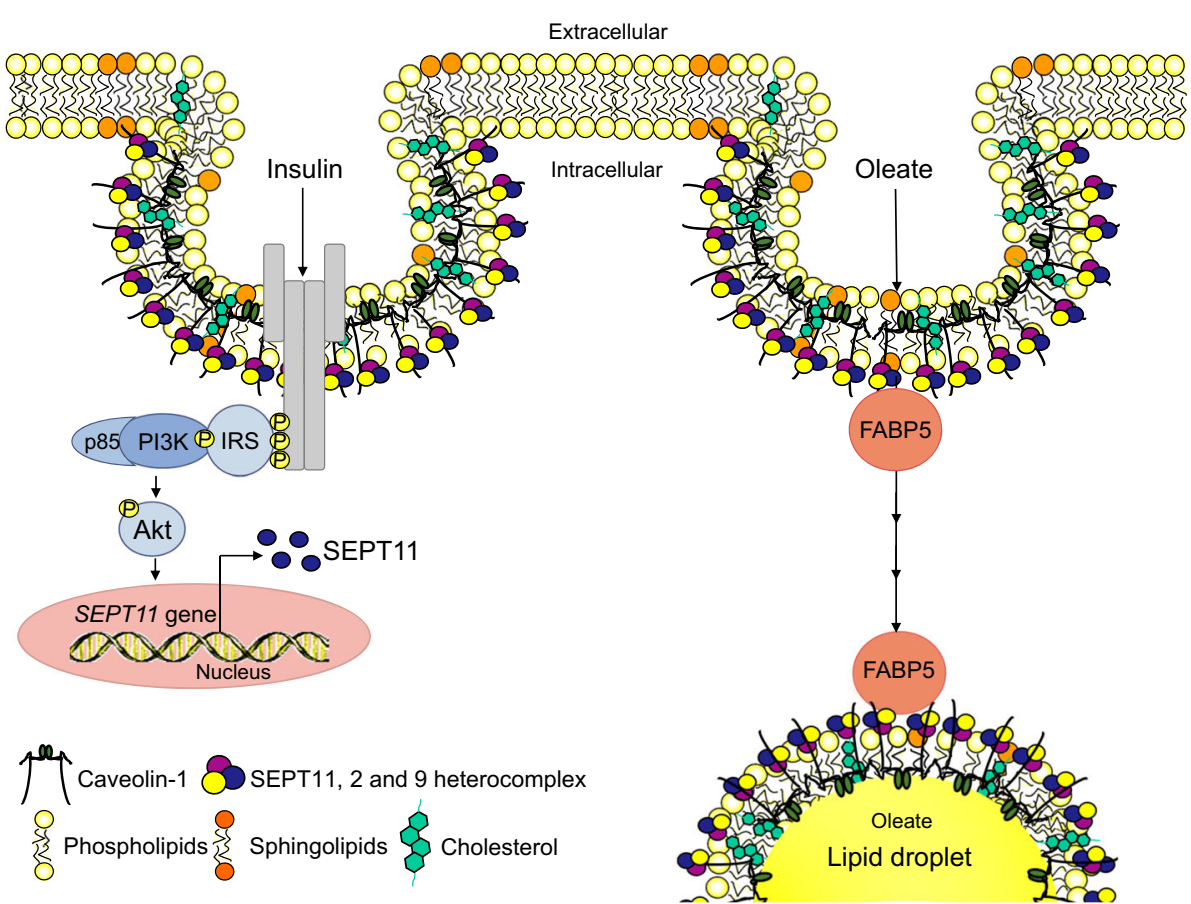


decreased activation of insulin signalling (i.e. Akt phosphorylation) in silenced 3T3-L1 cells. Our findings showing that the negative impact of SEPT11 downregulation on lipid accumulation was more dramatic in cells challenged with oleate, taken together with oleate-induced effects on SEPT11 intracellular distribution, suggest a link between SEPT11 and caveolae-to-LD transport of FA (Fig. 6). Once at the LD, SEPT11 may provide a platform to facilitate the compartmentalisation of CAV1 and/or FABP5. Interestingly, CAV1 has been also shown to modulate lipolysis by coupling protein kinase A activity to perilipin phosphorylation [37], and FABPs bind to and activate hormone sensitive lipase on treatment with oleate [44]. In accordance with these findings, we observed that oleate-induced lipolysis, an effect that has been previously reported in cells exposed to this FA [45], was reduced in Sept11-silenced adipocytes. In all, our data suggest a functional interaction between SEPT11, CAV1 and/or FABP5, which might be altered in obesity inasmuch as these three proteins followed different expression trends in the obese adipose tissue. Particularly, given our in vitro results, the increase in SEPT11 expression observed in subcutaneous and omental fat of obese individuals could represent an adaptive mechanism to facilitate insulin-mediated lipid storage in obese adipocytes, which seems nevertheless inefficient to improve the response of these cells to enhanced plasma insulin levels.

In summary, our data identify SEPT11 as a novel cytoskeletal component in adipocytes, wherein SEPT11 assembles into either filaments or rings depending on the stage of adipocyte differentiation and its selective interaction with other septins. Moreover, SEPT11 associates with CAV1 in adipocyte caveolae and, together with CAV1 and FABP5, binds to the LD surface on FA loading (Fig. 6). These results, together with our functional and expression studies, support a regulatory role of SEPT11 in lipid traffic and metabolism. Notably, this protein is increased in human obesity and related to adipocyte size in omental fat and to IR markers in subcutaneous fat. Taken together, these results broaden our understanding of the remodelling of the cytoskeleton in obesity development and IR and open new avenues for research on the control of lipid storage and metabolism in adipocytes.

Acknowledgements We gratefully acknowledge the valuable collaboration of L. Molero and all other members of the Adipobiology group (Instituto Maimónides de Investigación Biomédica de Córdoba/ University of Córdoba/Reina Sofia University Hospital, Spain), as well as E. Peralbo Santaella (Instituto Maimónides de Investigación Biomédica de Córdoba/University of Córdoba/Reina Sofia University Hospital, Spain), for their help and technical support, and of all members of the Department of Surgery and the Nutrition Unit of the Hospital Clínica Universidad de Navarra (Spain). We gratefully acknowledge W.S. Trimble (University of Toronto, Toronto, ON, Canada), K-I. Nagata (Institute for Developmental Research, Kasugai, Japan) and M. Kaczocha (Stony Brook University, Stony Brook, NY, USA) for kindly providing the plasmids coding for SEPT2 (phrGFPN1-SEPT2), SEPT9 (pEGFPC1-SEPT9), and FABP5 (pEGFPC1-FABP5). We also thank M.
Calzado (IMIBIC/Reina Sofia University Hospital/University of Córdoba, Spain) for the generous gift of the HEK-293 AD cell line and the reagents for the GST pull-down experiments.

Data availability Data are available on request from the authors.

Funding This work was funded by MINECO/FEDER (BFU201344229-R, AGL2012/39615; BFU2015-70454-REDT), Consejería de Salud y Bienestar Social/J. Andalucía/FEDER (PI-0200/2013), the Instituto de Salud Carlos III and Fondos FEDER (FIS PI12/00515, PI13/1430, PIE14/00005; co-funded by European Regional Development Fund/European Social Fund 'Investing in your future') and Plan de Investigación de la Universidad de Navarra (PIUNA) (2011-2014). CIBEROBN is an initiative of the Instituto de Salud Carlos III, Spain.

Duality of interest The authors declare that there is no duality of interest associated with this manuscript.

Contribution statement NM-C, AR, YR-R, AF-V and RV-M were responsible for acquisition of data. NM-C, AR, YR-R, AF-V, RV-M, JL-M, GF and MMM analysed data. GF and JL-M selected the patients. MMM, GF and AR designed the study. MMM and AR wrote the draft. MMM coordinated the study. All authors contributed to the interpretation of data and revision of the manuscript draft, and approved the final version. MMM is the guarantor of this work.

\section{References}

1. Spalding KL, Arner E, Westermark PO et al (2008) Dynamics of fat cell turnover in humans. Nature 453:783-787

2. Heinonen S, Saarinen L, Naukkarinen J et al (2014) Adipocyte morphology and implications for metabolic derangements in acquired obesity. Int J Obes (Lond) 38:1423-1431

3. Verstraeten VL, Renes J, Ramaekers FC et al (2011) Reorganization of the nuclear lamina and cytoskeleton in adipogenesis. Histochem Cell Biol 135:251-261

4. Yang W, Guo X, Thein S et al (2013) Regulation of adipogenesis by cytoskeleton remodelling is facilitated by acetyltransferase MEC17-dependent acetylation of alpha-tubulin. Biochem J 449:605-612

5. Yang W, Thein S, Wang X et al (2014) BSCL2/seipin regulates adipogenesis through actin cytoskeleton remodelling. Hum Mol Genet 23:502-513

6. Spiegelman BM, Farmer SR (1982) Decreases in tubulin and actin gene expression prior to morphological differentiation of 3T3 adipocytes. Cell 29:53-60

7. Lieber JG, Evans RM (1996) Disruption of the vimentin intermediate filament system during adipose conversion of 3T3-L1 cells inhibits lipid droplet accumulation. J Cell Sci 109:3047-3058

8. Kanzaki M, Pessin JE (2001) Insulin-stimulated GLUT4 translocation in adipocytes is dependent upon cortical actin remodeling. J Biol Chem 276:42436-42444

9. Ariotti N, Murphy S, Hamilton NA et al (2012) Postlipolytic insulin-dependent remodeling of micro lipid droplets in adipocytes. Mol Biol Cell 23:1826-1837

10. Kanzaki M, Pessin JE (2002) Caveolin-associated filamentous actin (Cav-actin) defines a novel F-actin structure in adipocytes. J Biol Chem 277:25867-25869 
11. Parton RG, del Pozo MA (2013) Caveolae as plasma membrane sensors, protectors and organizers. Nat Rev Mol Cell Biol 14:98112

12. Méndez-Giménez L, Rodríguez A, Balaguer I, Frühbeck G (2014) Role of aquaglyceroporins and caveolins in energy and metabolic homeostasis. Mol Cell Endocrinol 397:78-92

13. Mostowy S, Cossart P (2012) Septins: the fourth component of the cytoskeleton. Nat Rev Mol Cell Biol 13:183-194

14. Weirich CS, Erzberger JP, Barral Y (2008) The septin family of GTPases: architecture and dynamics. Nat Rev Mol Cell Biol 9: 478-489

15. Maimaitiyiming M, Kobayashi Y, Kumanogoh H, Nakamura S, Morita M, Maekawa S (2013) Identification of dynamin as a septin-binding protein. Neurosci Lett 534:322-326

16. Maimaitiyiming M, Kumanogoh H, Nakamura NK, Suzaki T, Maekawa S (2008) Biochemical characterization of membraneassociated septin from rat brain. J Neurochem 106:1175-1183

17. Moreno-Castellanos N, Guzmán-Ruiz N, Cano DA et al (2016) The effects of bariatric surgery-induced weight loss on adipose tissue in morbidly obese women depends on the initial metabolic status. Obes Surg 26:1757-1767

18. American Diabetes Association (2015) Classification and diagnosis of diabetes. Diabetes Care 38(Suppl):S8-S16

19. Rodríguez A, Gómez-Ambrosi J, Catalán Vet al (2012) The ghrelin O-acyltransferase-ghrelin system reduces TNF-alpha-induced apoptosis and autophagy in human visceral adipocytes. Diabetologia 55:3038-3050

20. Matthews DR, Hosker JP, Rudenski AS et al (1985) Homeostasis model assessment: insulin resistance and beta-cell function from fasting plasma glucose and insulin concentrations in man. Diabetologia 28:412-419

21. Guzman-Ruiz R, Ortega F, Rodriguez A et al (2014) Alarmin highmobility group B1 (HMGB1) is regulated in human adipocytes in insulin resistance and influences insulin secretion in beta-cells. Int $\mathrm{J}$ Obes (Lond) 38:1545-1554

22. Pulido MR, Diaz-Ruiz A, Jimenez-Gomez Y et al (2011) Rab18 dynamics in adipocytes in relation to lipogenesis, lipolysis and obesity. PLoS One 6:e22931

23. Vazquez-Martinez R, Martinez-Fuentes AJ, Pulido MR et al (2008) Rab18 is reduced in pituitary tumors causing acromegaly and its overexpression reverts growth hormone hypersecretion. J Clin Endocrinol Metab 93:2269-2276

24. Diaz-Ruiz A, Rabanal-Ruiz Y, Travez A et al (2013) The long coiled-coil protein NECC2 is associated to caveolae and modulates NGF/TrkA signaling in PC12 cells [corrected]. PLoS One 8:e73668

25. Hanai N, Nagata K, Kawajiri A et al (2004) Biochemical and cell biological characterization of a mammalian septin, Sept11. FEBS Lett 568:83-88

26. Huang YW, Yan M, Collins RF, Diciccio JE, Grinstein S, Trimble WS (2008) Mammalian septins are required for phagosome formation. Mol Biol Cell 19:1717-1726

27. Nagata K, Asano T, Nozawa Y, Inagaki M (2004) Biochemical and cell biological analyses of a mammalian septin complex, Sept7/9b/ 11. J Biol Chem 279:55895-55904
28. Watson RT, Shigematsu S, Chiang SH et al (2001) Lipid raft microdomain compartmentalization of TC10 is required for insulin signaling and GLUT4 translocation. J Cell Biol 154:829-840

29. Rodríguez A, Catalán V, Gómez-Ambrosi J, Frühbeck G (2007) Visceral and subcutaneous adiposity: Are both potential therapeutic targets for tackling the metabolic syndrome? Curr Pharm Des 13: 2169-2175

30. Li X, Serwanski DR, Miralles CP, Nagata K, De Blas AL (2009) Septin 11 is present in GABAergic synapses and plays a functional role in the cytoarchitecture of neurons and GABAergic synaptic connectivity. J Biol Chem 284:17253-17265

31. Gladfelter AS (2014) Cytoskeleton: cirque du septins. Curr Biol 24: R526-R528

32. Mavrakis M, Azou-Gros Y, Tsai FC et al (2014) Septins promote Factin ring formation by crosslinking actin filaments into curved bundles. Nat Cell Biol 16:322-334

33. Kinoshita M (2003) Assembly of mammalian septins. J Biochem 134:491-496

34. Cao L, Yu W, Wu Y, Yu L (2009) The evolution, complex structures and function of septin proteins. Cell Mol Life Sci 66:3309-3323

35. Peterson EA, Petty EM (2010) Conquering the complex world of human septins: implications for health and disease. Clin Genet 77: $511-524$

36. Cohen AW, Combs TP, Scherer PE, Lisanti MP (2003) Role of caveolin and caveolae in insulin signaling and diabetes. Am J Physiol Endocrinol Metab 285:E1151-E1160

37. Cohen AW, Razani B, Schubert W et al (2004) Role of caveolin-1 in the modulation of lipolysis and lipid droplet formation. Diabetes 53: 1261-1270

38. Hotamisligil GS, Bernlohr DA (2015) Metabolic functions of FABPs-mechanisms and therapeutic implications. Nat Rev Endocrinol 11:592-605

39. Furuhashi M, Hotamisligil GS (2008) Fatty acid-binding proteins: role in metabolic diseases and potential as drug targets. Nat Rev Drug Discov 7:489-503

40. Iso T, Maeda K, Hanaoka $\mathrm{H}$ et al (2013) Capillary endothelial fatty acid binding proteins 4 and 5 play a critical role in fatty acid uptake in heart and skeletal muscle. Arterioscler Thromb Vasc Biol 33: 2549-2557

41. Zimmerman AW, van Moerkerk HT, Veerkamp JH (2001) Ligand specificity and conformational stability of human fatty acid-binding proteins. Int J Biochem Cell Biol 33:865-876

42. Pilch PF, Meshulam T, Ding S, Liu L (2011) Caveolae and lipid trafficking in adipocytes. Clin Lipidol 6:49-58

43. Blouin CM, Le Lay S, Eberl A et al (2010) Lipid droplet analysis in caveolin-deficient adipocytes: alterations in surface phospholipid composition and maturation defects. J Lipid Res 51:945-956

44. Jenkins-Kruchten AE, Bennaars-Eiden A, Ross JR, Shen WJ, Kraemer FB, Bernlohr DA (2003) Fatty acid-binding proteinhormone-sensitive lipase interaction. Fatty acid dependence on binding. J Biol Chem 278:47636-47643

45. Xie W, Hamilton JA, Kirkland JL, Corkey BE, Guo W (2006) Oleate-induced formation of fat cells with impaired insulin sensitivity. Lipids 41:267-271 